\title{
Luxofracturas radiocarpianas: evaluación retrospectiva de una serie de casos
}

\author{
Ignacio Quinto Pages, Julián Parma, Ernesto Lombardo, Agustín Barbero, Emanuel González, \\ Jeremías Derico, Juan Manuel Baravalle, Manuel Vélez \\ Servicio de Ortopedia y Traumatología, Hospital de Emergencia “Clemente Álvarez”, Rosario, Santa Fe, Argentina
}

\begin{abstract}
RESUMEN
Introducción: Las luxofracturas radiocarpianas consisten en la pérdida total de contacto entre las superficies articulares de la primera fila del carpo y del radio. Se producen por traumatismos de alta energía. El objetivo de este estudio fue evaluar retrospectivamente una serie de casos para comparar la incidencia de estas lesiones, el manejo terapéutico y los resultados funcionales con la bibliografía publicada por centros de referencia en esta enfermedad. Materiales y Métodos: Entre febrero de 2018 y junio de 2020, se evaluó, en forma retrospectiva, a pacientes con luxofractura radiocarpiana, clasificada en tipos I y II según Dumontier. Criterios de inclusión: hombres y mujeres $>18$ años con luxofractura radiocarpiana cerrada o abierta y un seguimiento mínimo de 3 meses. Se llevó a cabo un seguimiento clínico/por imágenes mediante radiografías, el cuestionario DASH, la escala de muñeca modificada de la Clínica Mayo, la escala analógica visual para dolor y el PSQ-18. Resultados: Se evaluó a 8 pacientes con 9 lesiones, y un seguimiento promedio de 8 meses. Las luxaciones eran 4 tipo I y 5 tipo II. Todos fueron operados. Según valores finales, hubo 2 resultados excelentes, 6 buenos/aceptables y uno pobre. Conclusión: El mejor método para el tratamiento definitivo es quirúrgico. La correcta clasificación y el estudio de la enfermedad tienen un rol fundamental en la toma de decisiones terapéuticas.
\end{abstract}

Palabras clave: Luxación radiocarpiana; Dumontier; carpo; ligamentos radiocarpianos, estiloides radial; luxofractura.

Nivel de Evidencia: IV

\section{Radiocarpal Fracture-Dislocation: Retrospective Evaluation of a Case Series}

\begin{abstract}
Introduction: Radiocarpal fracture-dislocations consist of the total loss of contact between the articular surfaces of the first row of the carpus and the radius. They are caused by high-energy trauma. The purpose of the work is to retrospectively evaluate a series of cases to compare the incidence of these lesions, their therapeutic management and functional outcomes with the literature published by reference centers in this pathology. Materials and Methods: Between February 2018 and June 2020 we retrospectively evaluated patients with radiocarpal fracture-dislocations, which were classified into groups I and II according to Dumontier. Inclusion criteria: males and females over 18 years of age with closed or open radiocarpal fracture-dislocations with a minimum follow-up of 3 months. A clinical/imaging follow-up was carried out using radiographs, the DASH questionnaire (Disabilities of the Arm, Shoulder and Hand), Modified Mayo Wrist Score, visual analog scale (VAS) and Patient Satisfaction Questionnaire Short Form (PSQ-18). Results: Eight patients with nine lesions with an average follow-up of 8 months were evaluated. Lesions were grouped into type I (4) and type II (5). All were surgically operated. According to the final values, outcomes were excellent in two cases, good/acceptable in six, and poor in one. Conclusion: We believe that the best method for definitive treatment is surgical. The correct classification and study of the pathology will play a fundamental role in making therapeutic decisions.
\end{abstract}

Keywords: Radiocarpal luxation; Dumontier; carpus; radiocarpal ligaments; radial styloid; fracture-dislocation. Level of Evidence: IV

\footnotetext{
Recibido el 13-5-2021. Aceptado luego de la evaluación el 12-8-2021 • Dr. IGNACIO QUINTO PAGES • ignaqp@ @otmail.com https://orcid.org/0000-0002-6176-2135 Cómo citar este artículo: Quinto Pages I, Parma J, Lombardo E, Barbero A, González E, Derico J, Baravalle JM, Vélez M. Luxofracturas radiocarpianas: evaluación retrospectiva de una serie de casos. Rev Asoc Argent Ortop Traumatol 2021;86(5):629-638. https://doi.org/10.15417/issn. 1852-7434.2021.86.5.1373
} 


\section{INTRODUCCIÓN}

Las luxofracturas radiocarpianas se definen como la pérdida total de contacto entre las superficies articulares de la primera fila del carpo (escafoides y semilunar) y del radio, con desplazamientos principalmente hacia volar o dorsal. Además, se pueden detectar lesiones asociadas en la zona de la muñeca o las articulaciones proximales. ${ }^{1,2}$

Se producen en el contexto de traumatismos de alta energía. No hay consenso en cuanto al mecanismo de la lesión, se estima que se debe a una combinación de hiperextensión, pronación y desviación radial de la muñeca, aunque algunos autores lo asocian a mecanismos en flexión. Se trata de lesiones raras, con una frecuencia de entre el $0,2 \%$ y el $6 \%$ de todas las lesiones radiocarpianas. ${ }^{1-3}$ La clasificación utilizada es la propuesta por Dumontier que divide a estas lesiones en dos grupos. ${ }^{1-4}$

Hay acuerdo en que el manejo de este cuadro en la etapa aguda se realiza en dos tiempos: primero, la reducción (cerrada o abierta, de ser necesario) y, segundo, la cirugía definitiva. ${ }^{1-4}$ Respecto al método quirúrgico empleado, la bibliografía es contradictoria. ${ }^{1-3}$

El objetivo de este estudio fue evaluar retrospectivamente una serie de casos de nuestro Servicio para comparar tanto la incidencia de estas lesiones como el manejo terapéutico y los resultados funcionales con la bibliografía publicada por centros de referencia mundial en esta enfermedad.

\section{MATERIALES Y MÉTODOS}

Se llevó a cabo un estudio retrospectivo de pacientes tratados entre febrero de 2018 y junio de 2020. Los criterios de inclusión fueron: hombres y mujeres $>18$ años con luxofractura radiocarpiana cerrada o abierta diagnosticada y clasificada mediante radiografías y con un seguimiento mínimo de tres meses. Los criterios de exclusión fueron: fracturas tipo Barton (son las únicas lesiones de muñeca que se pueden malinterpretar como Dumontier; sin embargo, las diferencia la ausencia de incongruencia de la carilla articular), no contar con radiografías al ingresar que documenten la luxación, falta de controles posoperatorios.

En la sala de emergencia, se tomaron radiografías anteroposteriores y de perfil de muñeca o antebrazo (Figura 1). Se registraron la edad, el sexo, el mecanismo de producción y las lesiones asociadas. Un paciente tenía una fractura del pilón tibial; otro, una fractura de fémur y tibia diafisaria, y otro, una luxofractura de Monteggia, lesión de partes blandas tipo herida con colgajo en muslo, fractura de falange del pie, segundo metacarpiano homolateral y traumatismo craneoencefálico grave que requirió el ingreso en la Unidad de Cuidados Intensivos por 14 días (Tabla 1).
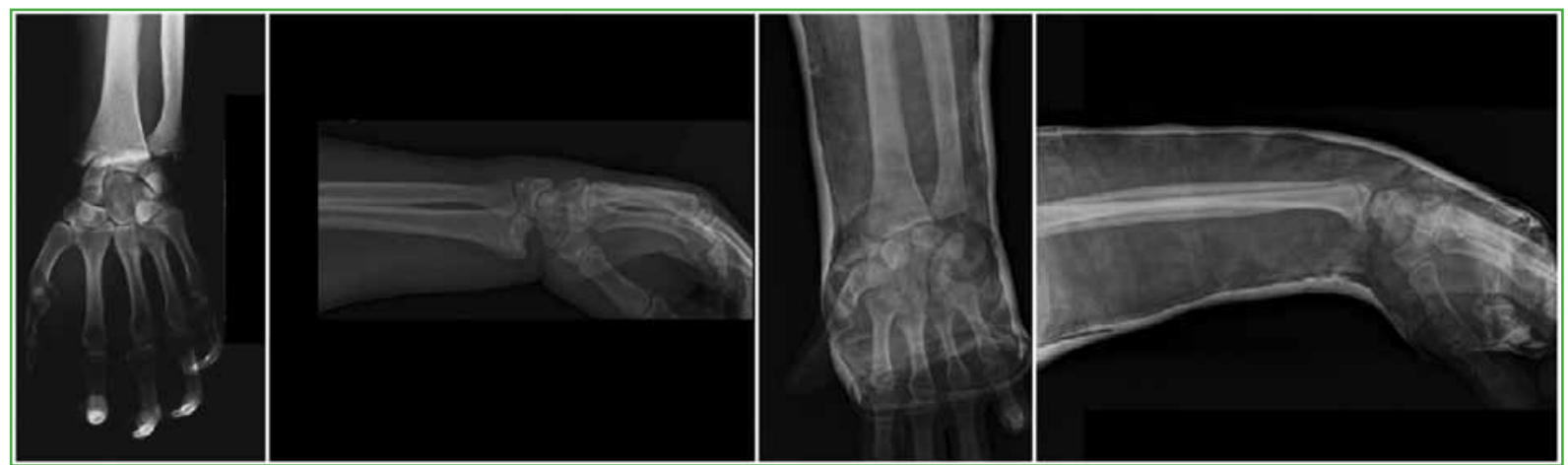

Figura 1. Caso 3. Radiografías anteroposteriores y de perfil de muñeca al ingresar y luego de la reducción. 
Tabla 1. Datos de los pacientes

\begin{tabular}{|c|c|c|c|c|c|c|c|c|}
\hline Caso & Sexo & Edad & Dumontier & $\begin{array}{l}\text { Expo- } \\
\text { sición }\end{array}$ & Mecanismo & $\begin{array}{c}\text { Reducción/inmovilización } \\
\text { en etapa aguda }\end{array}$ & $\begin{array}{l}\text { Lesiones } \\
\text { asociadas }\end{array}$ & $\begin{array}{c}\text { Miembro } \\
\text { dominante }\end{array}$ \\
\hline 1 & $\mathrm{~F}$ & 46 & II & No & $\begin{array}{l}\text { No recuerda } \\
\quad(\text { AVP })\end{array}$ & $\begin{array}{c}\text { Cerrada/ } \\
\text { Yeso braquipalmar }\end{array}$ & No & Sí \\
\hline 2 & M & 33 & I & No & $\begin{array}{l}\text { Extensión } \\
\text { (AVP) }\end{array}$ & $\begin{array}{c}\text { Cerrada/ } \\
\text { Yeso braquipalmar }\end{array}$ & $\begin{array}{l}\text { "Scalp" } \\
\text { en rodilla }\end{array}$ & Sí \\
\hline 3 & M & 20 & I & No & $\begin{array}{c}\text { No recuerda } \\
\text { (caída de altura) }\end{array}$ & $\begin{array}{c}\text { Cerrada/ } \\
\text { Yeso braquipalmar }\end{array}$ & No & Sí \\
\hline 4 & $\mathrm{~F}$ & 47 & II & GI & $\begin{array}{l}\text { No recuerda } \\
\text { (AVP) }\end{array}$ & $\begin{array}{l}\text { Cerrada + ATB IV/ } \\
\text { Yeso braquipalmar }\end{array}$ & No & No \\
\hline 5 & M & 21 & I & No & $\begin{array}{l}\text { Extensión } \\
\text { (AVP) }\end{array}$ & $\begin{array}{l}\text { Cerrada/ } \\
\text { Yeso braquipalmar }\end{array}$ & $\begin{array}{c}\text { Fracturas } \\
\text { de fémur/tibia } \\
\text { y peroné }\end{array}$ & Sí \\
\hline 6 & M & 25 & I & No & $\begin{array}{c}\text { Extensión (caída } \\
\text { de altura) }\end{array}$ & $\begin{array}{c}\text { Cerrada/ } \\
\text { Yeso braquipalmar }\end{array}$ & $\begin{array}{c}\text { Fractura del } \\
\text { pilón tibial }\end{array}$ & Sí \\
\hline 7 & M & 30 & II & No & $\begin{array}{l}\text { No recuerda } \\
(\mathrm{AVP})\end{array}$ & $\begin{array}{c}\text { Cerrada/ } \\
\text { Yeso braquipalmar }\end{array}$ & $\begin{array}{l}\text { Luxofractura } \\
\text { de Monteggia, } \\
\text { fractura } 2^{\circ} \text {. } \\
\text { MTC, "scalp" } \\
\text { en muslo, } \\
\text { TCE grave }\end{array}$ & No \\
\hline 8 & M & 29 & II & No & $\begin{array}{l}\text { Extensión } \\
\text { (AVP) }\end{array}$ & $\begin{array}{c}\text { Cerrada/ } \\
\text { Yeso braquipalmar }\end{array}$ & No & Sí \\
\hline 9 & M & 29 & II & No & $\begin{array}{l}\text { Extensión } \\
\text { (AVP) }\end{array}$ & $\begin{array}{c}\text { Cerrada/ } \\
\text { Yeso braquipalmar }\end{array}$ & No & No \\
\hline \multirow{4}{*}{$\begin{array}{l}\text { Pro- } \\
\text { me- } \\
\text { dios }\end{array}$} & $\begin{array}{c}\mathrm{M} \\
75 \%\end{array}$ & 31.1 & I $44,5 \%$ & $\begin{array}{l}\text { No } \\
88,9 \%\end{array}$ & $\begin{array}{c}\text { No recuerda } \\
44,4 \%\end{array}$ & Cerrada/Yeso $88,9 \%$ & No $55,5 \%$ & Sí $66,6 \%$ \\
\hline & $\begin{array}{c}\mathrm{F} \\
25 \%\end{array}$ & & II $55,5 \%$ & $\begin{array}{c}\text { GI } \\
11,1 \%\end{array}$ & $\begin{array}{l}\text { Extensión } \\
55,6 \%\end{array}$ & $\begin{array}{l}\text { Cerrada + ATB IV/ } \\
\text { Yeso } 11,1 \%\end{array}$ & $\begin{array}{c}\text { Lesión } \\
\text { homolateral } \\
11,1 \%\end{array}$ & No $33,3 \%$ \\
\hline & & & & & AVP $77,7 \%$ & & $\begin{array}{c}\text { Otras lesiones } \\
44,4 \%\end{array}$ & \\
\hline & & & & & $\begin{array}{c}\text { Caída de altura } \\
22,3 \%\end{array}$ & & & \\
\hline
\end{tabular}

$\mathrm{M}=$ masculino, $\mathrm{F}=$ femenino, $\mathrm{GI}=$ Gustillo-Anderson, $\mathrm{AVP}=$ accidente en la vía pública, $\mathrm{ATB}$ IV = antibiótico intravenoso, $\mathrm{MTC}=$ metacarpiano, $\mathrm{TCE}=$ traumatismo craneoencefálico.

Se analizaron las lesiones de cada paciente y se formaron dos grupos diferenciados según la clasificación de Dumontier (Tabla 2). Se practicó una reducción cerrada en la sala de emergencia bajo anestesia local mediante tracción axial y desviación dorsal/palmar según la luxación e inmovilización con yeso braquipalmar. Posteriormente, se solicitó una tomografía computarizada (Figura 2) de cortes finos, axiales, coronales y sagitales con reconstrucción 3D para la planificación quirúrgica. Si las fracturas eran expuestas, se las trató dentro de las tres horas de producidas bajo el protocolo infectológico del hospital según la clasificación de Gustillo-Anderson. A los pacientes con exposición tipo I, se les administró cefalotina por vía intravenosa, durante $24 \mathrm{~h}$, y a aquellos con exposición tipos II y III, clindamicina más gentamicina por 72 h, además de una limpieza quirúrgica. 
Tabla 2. Clasificación de las luxofracturas radiocarpianas según Dumontier (2001)

\begin{tabular}{|l|l|l|}
\hline Clasificación & Lesión & Tratamiento \\
\hline Grupo I & $\begin{array}{l}\text { Luxación de la articulación radiocarpiana pura } \\
\text { + avulsión cortical/fractura de la punta de la } \\
\text { estiloides radial }\end{array}$ & $\begin{array}{l}\text { Reparación de ligamentos radio-escafo-grande + radio- } \\
\text { lunar largo vía palmar } \\
\text { La avulsión dorsal no se trata, ya que se considera una } \\
\text { lesión capsuloperióstica tipo Bankart más que una } \\
\text { rotura de los ligamentos dorsales }\end{array}$ \\
\hline Grupo II & $\begin{array}{l}\text { Luxación de la articulación radiocarpiana + } \\
\text { fractura de la estiloides radial de, al menos, un } \\
\text { tercio de la fosa escafoidea }\end{array}$ & $\begin{array}{l}\text { Osteosíntesis del fragmento de la estiloides radial por } \\
\text { vía dorsal }\end{array}$ \\
\hline
\end{tabular}

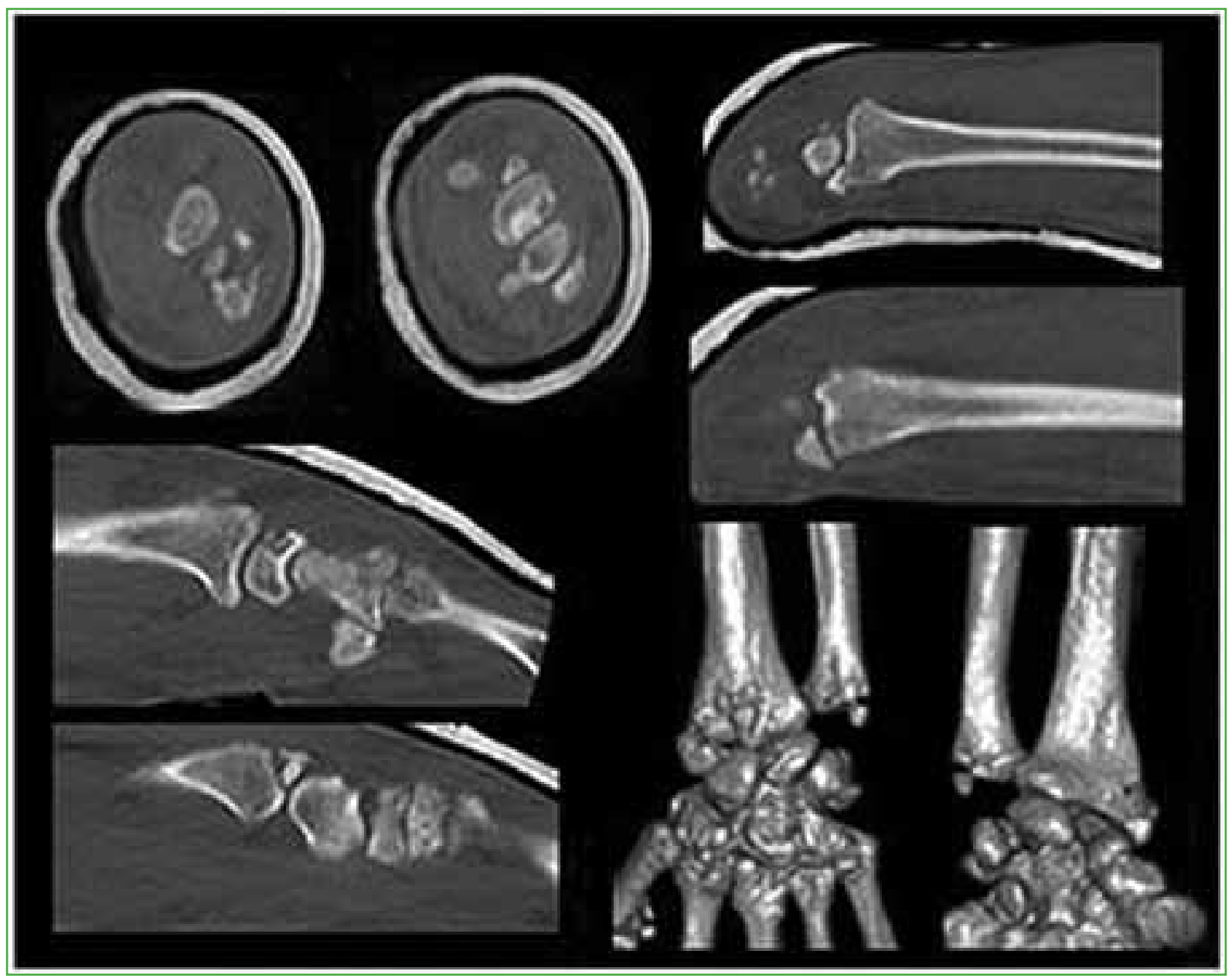

Figura 2. Caso 3. Tomografía computarizada de muñeca después de la reducción.

Con relación a la técnica quirúrgica, en su gran mayoría, este tipo de lesiones requiere de una osteosíntesis de la estiloides radial, reanclado de la cápsula articular y sutura ligamentaria (radiosemilunar corto). Se utilizó el abordaje de Henry para radio distal (Figura 3). No fueron necesarios abordajes dorsales debido a la correcta reducción indirecta de los fragmentos fracturarios. La estabilidad/reducción fue controlada mediante radioscopia dinámica en el quirófano (Figura 4). 


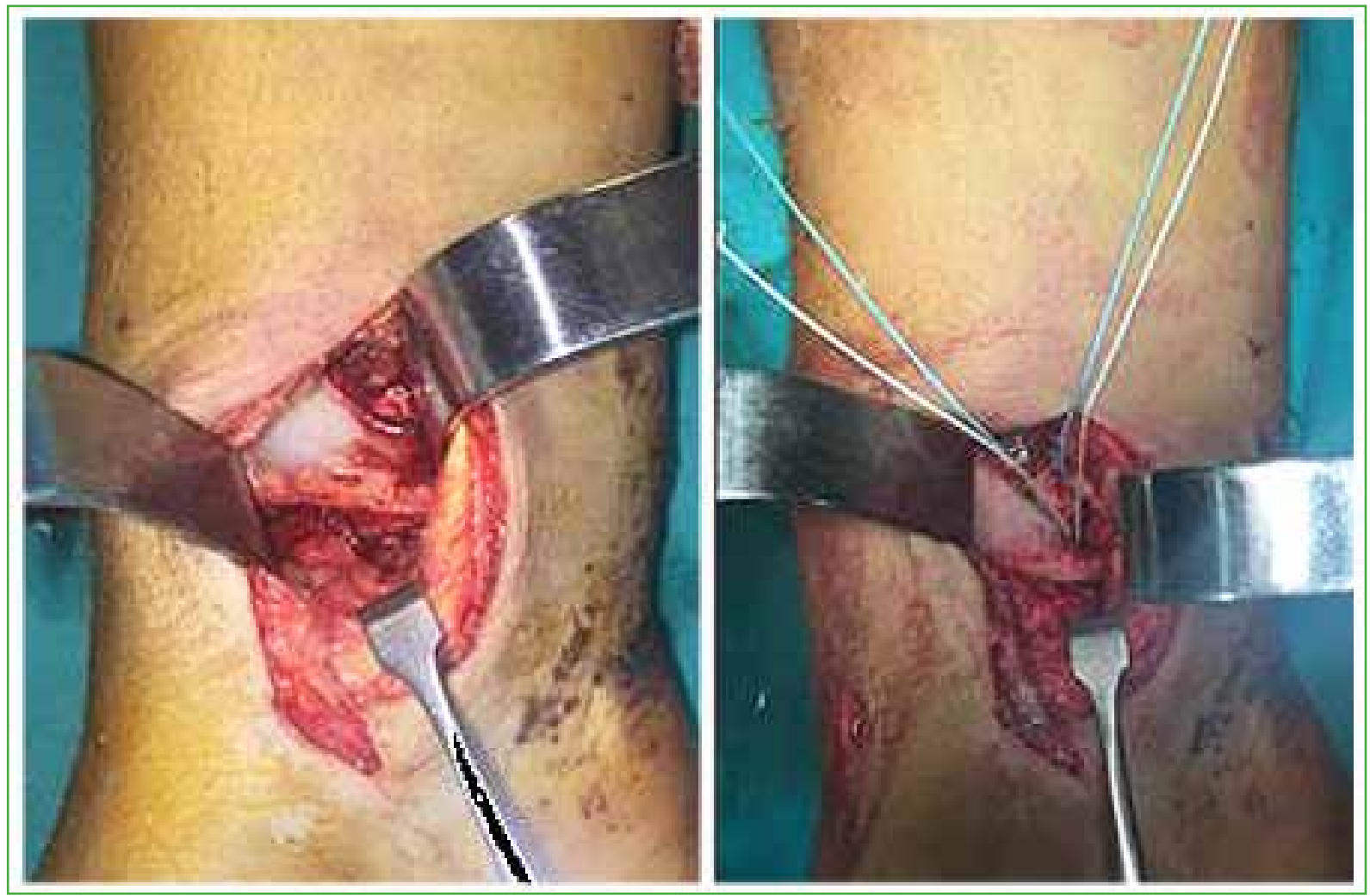

Figura 3. Caso 3. Imágenes intraoperatorias. Abordaje de Henry modificado.
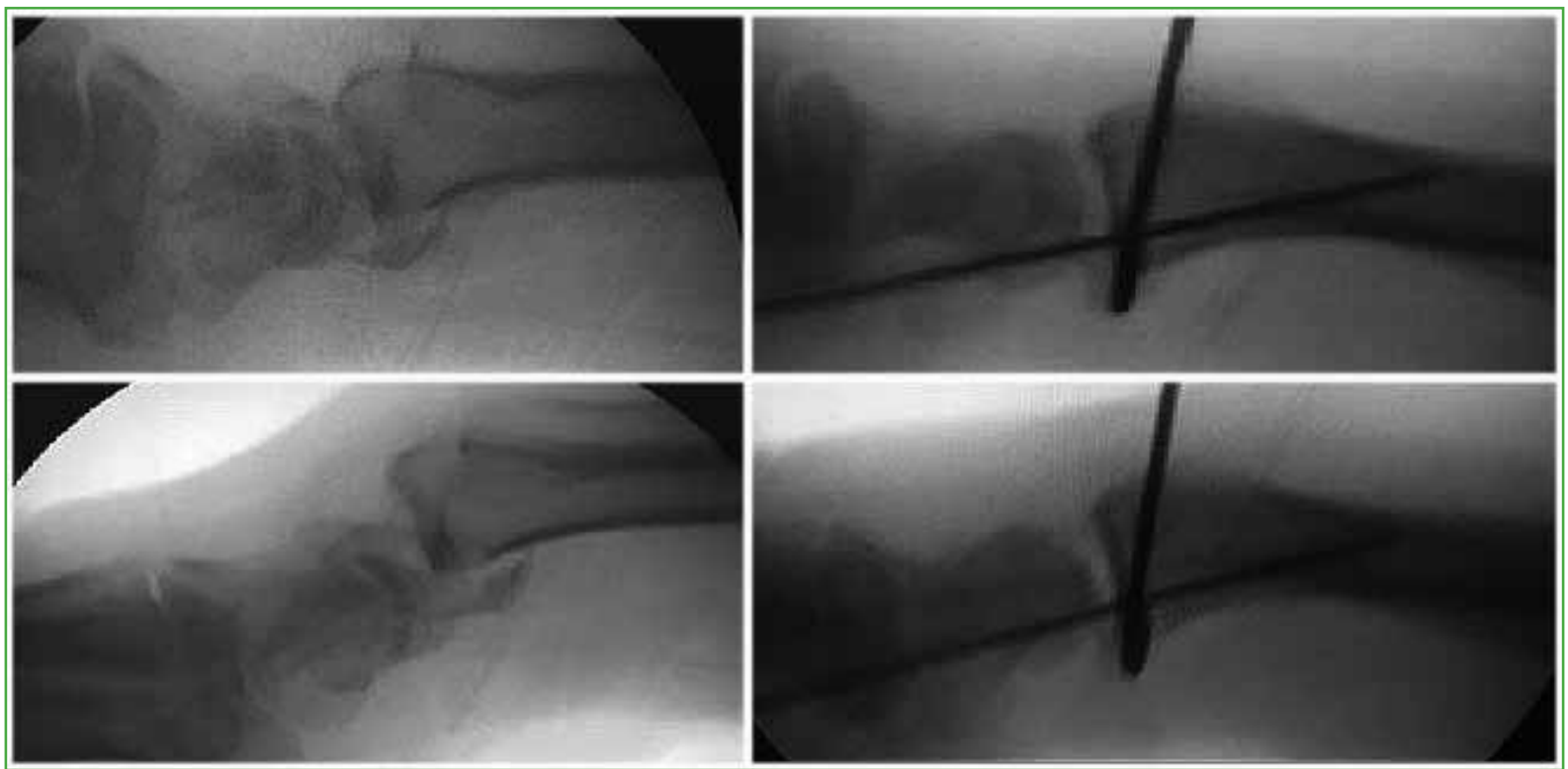

Figura 4. Caso 9. Imágenes obtenidas con el intensificador en el quirófano. 
Por decisión del cirujano tratante, dos pacientes fueron inmovilizados después de la cirugía con un yeso antebraquipalmar y siete, con férula antebraquipalmar durante un promedio de 30 días (rango 28-45). A los 30 días en promedio, se repitieron los estudios por imágenes, y se retiró la inmovilización para comenzar con movimientos pasivos y activos guiados por profesionales. No se hallaron diferencias en cuanto al método de inmovilización utilizado. Los elementos de osteodesis se retiraron a las 4-6 semanas, dos pacientes tuvieron intolerancia cutánea local que desapareció al retirar la inmovilización.

Los controles se realizaron a los 15, 30, 90, 180 y 345 días con radiografías anteroposteriores y de perfil de muñeca (Figuras 5 y 6). Desde el punto de vista objetivo clínico/funcional, se evaluaron los rangos de movilidad en flexión, extensión, pronosupinación y las desviaciones cubital y radial, tomando como parámetro normal de referencia el miembro contralateral sano en una escala del $0 \%$ al $100 \%$.

Como complemento de la evaluación subjetiva, se empleó el cuestionario DASH y la escala de muñeca modificada de la Clínica Mayo. El grado de dolor se determinó con la escala analógica visual $(0=$ sin dolor, $10=$ dolor máximo). El nivel de satisfacción del paciente se determinó mediante el Patient Satisfaction Questionnaire Short Form (PSQ-18) en muy satisfecho, satisfecho, poco satisfecho e insatisfecho. Se buscaron signos de falta de consolidación (retraso de la consolidación/seudoartrosis), artrosis y alteraciones asociadas a la articulación radiocubital distal.
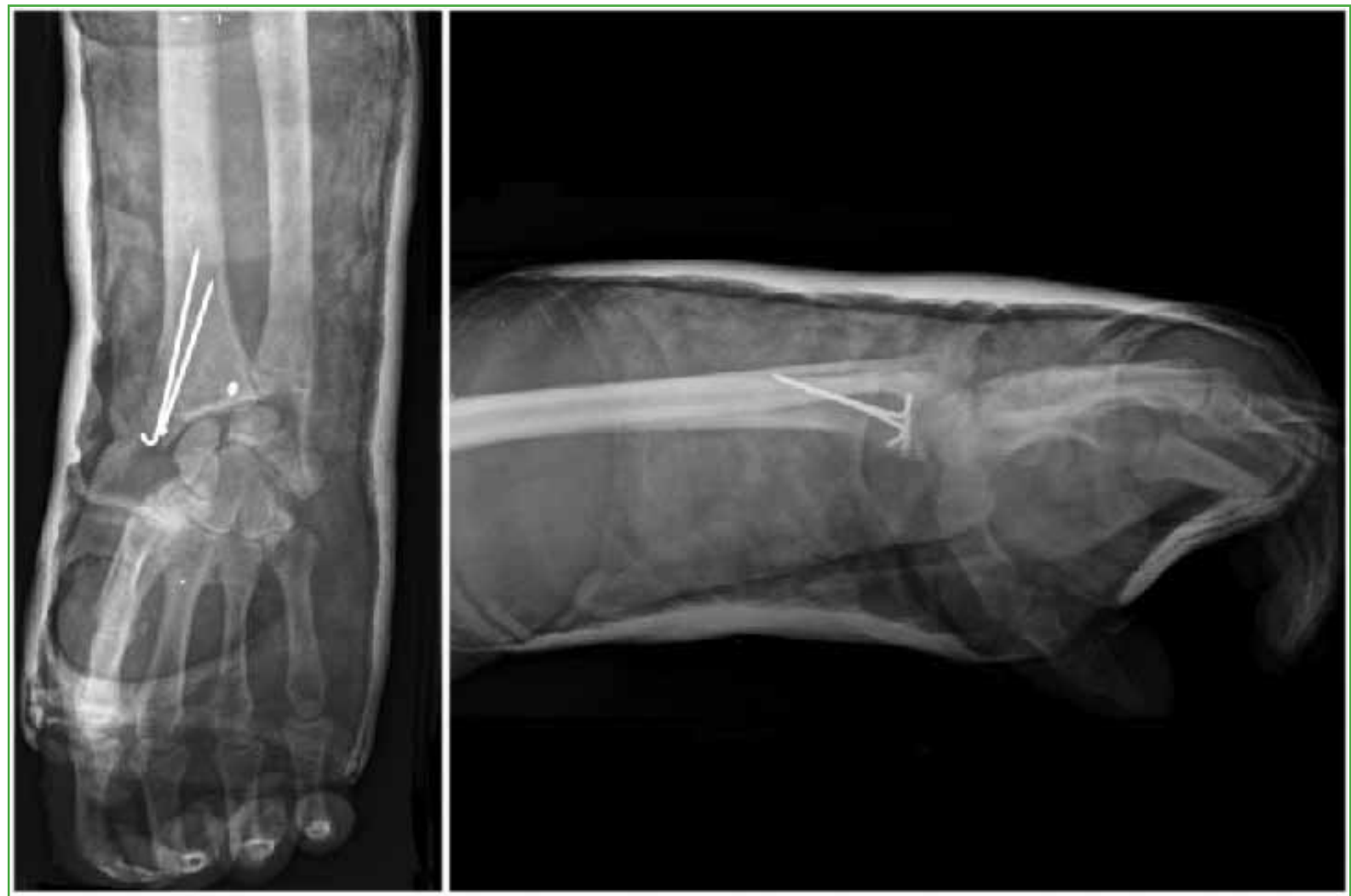

Figura 5. Caso 3. Radiografías anteroposterior y de perfil de muñeca en el posoperatorio inmediato. 


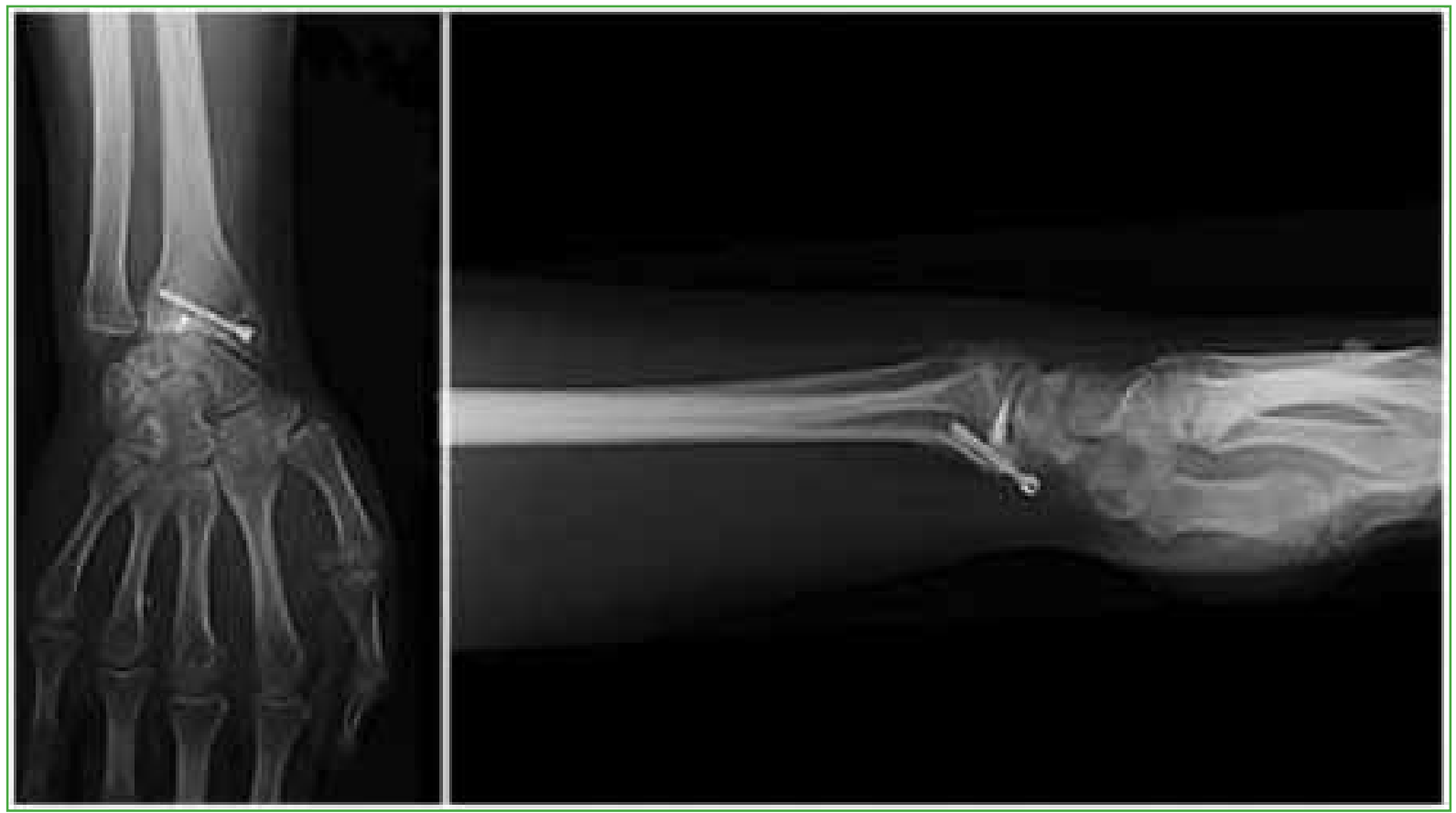

Figura 6. Caso 5. Radiografías anteroposterior y de perfil de muñeca a los 15 meses de la cirugía.

\section{RESULTADOS}

Se evaluó una serie retrospectiva de ocho pacientes con nueve luxofracturas radiocarpianas (una bilateral). Hubo un predominio del sexo masculino (75\%). El promedio de edad era de 31.1 años (rango 20-47).

El mecanismo de lesión involucró caídas de altura ( 2 casos) o accidentes en la vía pública ( 3 casos) con muñeca en extensión; el resto de los pacientes no recuerda la mecánica, aunque se tratan de accidentes de alta energía.

Cuatro luxaciones fueron tipo I de Dumontier, con desplazamientos hacia volar, mientras que cinco fueron tipo II (4 con desplazamientos volares y 1 dorsal; un paciente tenía una lesión bilateral, ambas hacia volar).

El tiempo promedio de evolución de las nueve luxofracturas radiocarpianas hasta la cirugía fue de 5.3 días (rango 3-11). Dada nuestra experiencia con el abordaje de Henry modificado para radio distal, se utilizó en ocho casos y no fue necesario un abordaje dorsal. En un paciente, la reducción cerrada fue aceptable y se decidió la fijación mediante tratamiento percutáneo.

En seis oportunidades, se utilizó una placa palmar para la reducción y el sostén de la carilla articular. En contrapartida, tres pacientes requirieron solo osteodesis a nivel de la estiloides radial y uno necesitó una doble fijación (mediante osteodesis y osteosíntesis) para lograr una mayor estabilidad, sumado a un tornillo de Herbert interfragmentario en busca de simplificar un trazo conminuto. Cabe mencionar que, en tres casos, se emplearon arpones como método de reparación de la cápsula articular en combinación con la fijación de la estiloides radial, sin requerimiento de placa palmar y, en tres casos, fue necesaria la reparación mediante la sutura del ligamento radiosemilunar corto.

Una de las luxofracturas era expuesta (tipo I de Gustillo-Anderson) y fue tratada según protocolo. Ningún paciente tuvo déficit sensitivo-motor ni afección vascular asociada.

Con un seguimiento promedio de ocho meses (rango 3-12), la evaluación del rango de movilidad de flexión, extensión y pronosupinación y las desviaciones cubital y radial de manera comparativa con el miembro contralateral (Figura 7) (salvo el paciente con lesión bilateral en quien se evaluó cada lesión por separado) determinó que cuatro pacientes tenían un rango de movilidad del 75-99\%; cuatro, del 50-74\% y uno, del 25-49\%, respectivamente. Con los datos recabados de esta manera, podemos establecer que dos pacientes tuvieron resultados excelentes (91$100 \%)$; seis, buenos/aceptables $(65-90 \%)$ y uno, pobre $(<65 \%)$. 

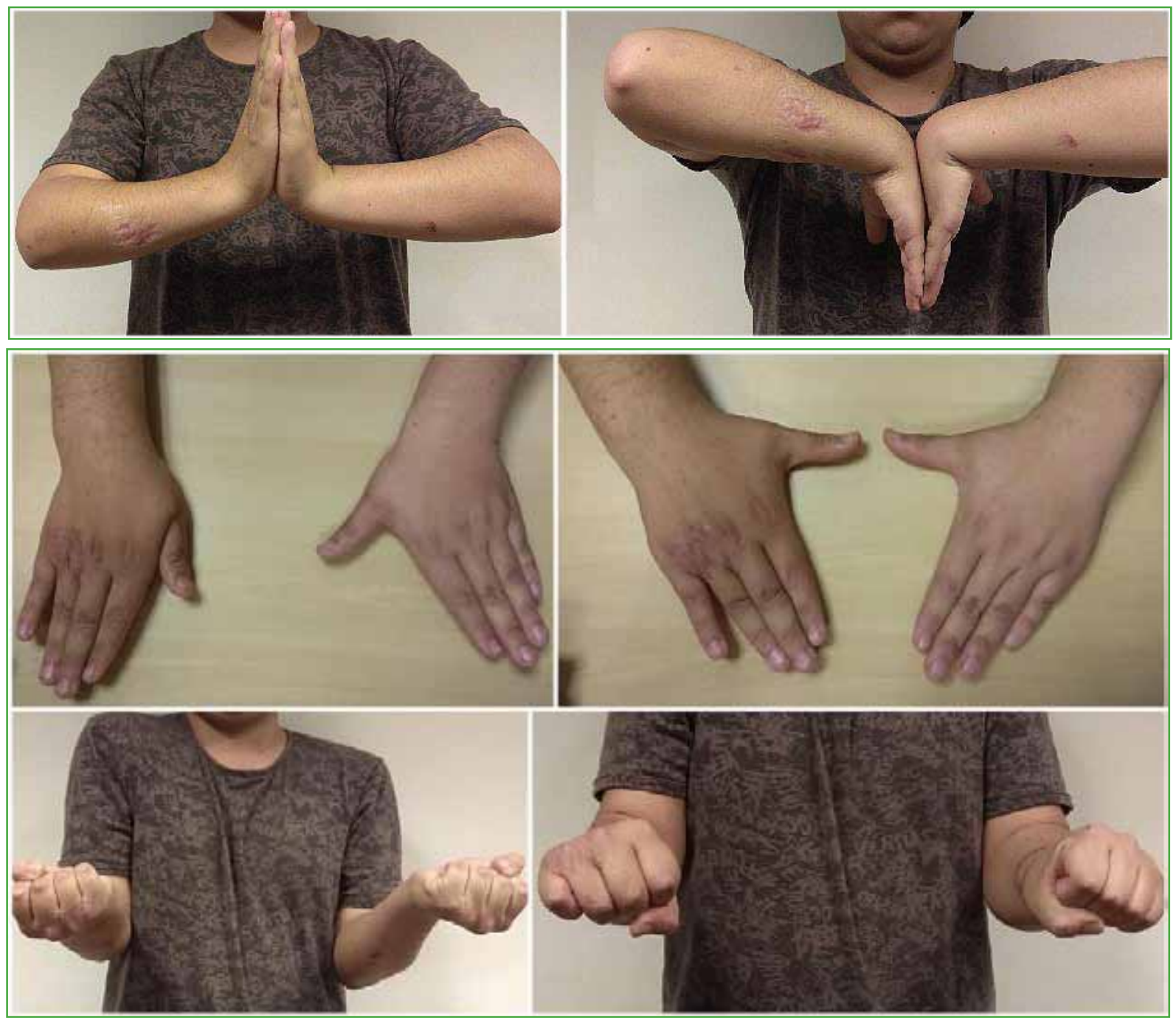

Figura 7. Caso 3. Resultados funcionales a los 12 meses de la cirugía. Rango de movilidad del 75-99\%, según la escala de muñeca modificada de la Clínica Mayo.

La evaluación subjetiva respecto al dolor según la escala analógica visual arrojó un promedio de 1,2 (rango 0-3) en reposo y 3,8 (rango 0-8) en actividad. Los resultados constatados al final del seguimiento mediante el cuestionario DASH y la escala de muñeca modificada de la Clínica Mayo se muestran en la Tabla 3. Por último, el nivel de satisfacción según el PSQ-18 fue muy satisfecho (2 pacientes), satisfecho (5 casos) y el restante, insatisfecho, aunque era capaz de realizar tareas básicas/cotidianas.

\section{DISCUSIÓN}

Se realizó un estudio retrospectivo en el que se evaluó a una serie de pacientes con luxofracturas radiocarpianas. En línea con la bibliografía, este tipo de lesiones son infrecuentes, tienen una incidencia de 4,5 pacientes por año, en nuestro Servicio. ${ }^{5}$ En la bibliografía, se comunican el predominio de mecanismos de producción de alta energía, la prevalencia del sexo masculino y la mayor incidencia de lesiones en la tercera década de la vida, información similar a la hallada en este estudio. , $2,3,5^{-1}$

A diferencia de la mayoría de los estudios que comunican un predominio de desplazamiento hacia dorsal, nuestros casos presentaron un predominio volar. ${ }^{1,2}$ La clasificación y la estadificación sistemáticas de esta enfermedad permiten orientar y facilitar la planificación del tratamiento..$^{1-3}$ 
Tabla 3. Resultados clínico-funcionales

\begin{tabular}{|c|c|c|c|c|c|c|}
\hline Caso & $\begin{array}{c}\text { Rehabilitación } \\
\text { (días de posoperatorio) }\end{array}$ & Movilidad (\%) & $\begin{array}{l}\text { Puntaje de la } \\
\text { Clínica Mayo }\end{array}$ & DASH & EAV (dolor) & $\begin{array}{l}\text { EAV (dolor } \\
\text { en actividad) }\end{array}$ \\
\hline 1 & 30 & $90 \%$ & Bueno (85) & $11,6 \%$ & 1 & 2 \\
\hline 2 & 28 & $95 \%$ & Excelente (92) & $7,5 \%$ & 0 & 1 \\
\hline 3 & 33 & $85 \%$ & Bueno (80) & $15 \%$ & 1 & 3 \\
\hline 4 & 40 & $70 \%$ & Malo (75) & $23,3 \%$ & 2 & 7 \\
\hline 5 & 30 & $60 \%$ & Malo(70) & $20,8 \%$ & 2 & 7 \\
\hline 6 & 35 & $99 \%$ & Excelente (95) & $9,9 \%$ & 0 & 0 \\
\hline 7 & 35 & $35 \%$ & Pobre (60) & $65,8 \%$ & 3 & 8 \\
\hline 8 & 42 & $70 \%$ & Bueno (80) & $16,6 \%$ & 1 & 4 \\
\hline 9 & 28 & $70 \%$ & Bueno (85) & $15,8 \%$ & 1 & 3 \\
\hline \multirow[t]{3}{*}{ Promedios } & Sí 100\% & Leve $66,6 \%$ & Excelente $22,2 \%$ & $20,7 \%$ & 1,2 & 3,8 \\
\hline & 33.4 días & Moderado 22,2\% & Bueno $44,4 \%$ & & & \\
\hline & & Alto $11,1 \%$ & Malo 22,2\% & & & \\
\hline
\end{tabular}

Pobre $11 \%$

$\mathrm{EAV}=$ escala analógica visual.

En los estudios presentados por Dumontier, es importante la reparación de los ligamentos radiocarpianos (por sutura directa o arpones) para evitar el desplazamiento cubital del carpo, así como también la inestabilidad residual. ${ }^{1-3}$ Propone abordar por palma las lesiones tipo I asociando la colocación de alambres de Kirschner a la estiloides radial para aumentar la estabilidad, en contraposición con las tipo II en las que aconseja un abordaje por dorsal garantizando la reducción anatómica.

Como señala Cornu, la estabilización con clavijas de Kirschner complementada con una inmovilización de 4 a 6 semanas son medidas suficientes para lograr la cicatrización ligamentaria. ${ }^{6}$ En nuestra institución, los abordajes se realizaron por palma mediante la vía de Henry modificada, no hubo complicaciones asociadas, se le asignó una importancia principal a la fijación de la estiloides radial para la estabilidad de los ligamentos radiolunar largo y radioseminular corto.

Potter y cols. proponen la colocación de una placa puente radiocarpiana complementada con ligamentoplastia mediante un abordaje dorsal. Los autores obtuvieron buenos resultados, aunque sin un seguimiento que permita establecer conclusiones finales sobre esta técnica.

En los reportes de Spiry, se establece la necesidad de complementar el método de fijación seleccionado con clavijas de Kirschner para aquellas fracturas conminutas. Además, comunica que seis pacientes requirieron una artrodesis radiocarpiana. ${ }^{4}$ En nuestro estudio, no fue necesario volver a operar a ningún paciente.

No se hallaron signos de osteoartritis. Diversos autores afirman que se trata de una complicación frecuente en los pacientes con lesiones intracarpianas asociadas sin reparación, y que aparecen aproximadamente a los 24 meses de la cirugía. ${ }^{1-3}$ Para diagnosticarlas, Spiry y Girard proponen utilizar la radioscopia dinámica intraoperatoria, el artroscopio o la tomografía computarizada posoperatoria. ${ }^{4,5}$ 
Las limitaciones de nuestro estudio son el diseño retrospectivo y el acotado número de la muestra, junto con la heterogénea terapéutica utilizada debido a la baja incidencia de este cuadro. También el escaso tiempo de seguimiento, especialmente en algunos pacientes, aunque de mínima suficiente para lograr los objetivos propuestos; sin embargo, un seguimiento más prolongado ayudaría a seguir documentando hallazgos clínicos y por imágenes (sobre todo, aquellos de carácter crónico). Cabe mencionar, no obstante, que esto se ve condicionado principalmente por la dificultad de contacto y acceso de nuestra población al efector. Consideramos como principal fortaleza el abordaje de un cuadro traumático poco frecuente. Se plantea la posibilidad de llevar a cabo un estudio prospectivo, aleatorizado y multicéntrico con más casos y un seguimiento más prolongado para poder documentar la evolución de esta compleja enfermedad y sus complicaciones.

\section{CONCLUSIONES}

Las luxofracturas radiocarpianas son lesiones raras asociadas a traumatismos de alta energía; el manejo inicial requiere de un diagnóstico preciso y precoz, seguido de la reducción y una tomografía computarizada para evaluar mejor la lesión. El tratamiento es quirúrgico, y la clasificación y el estudio correctos son fundamentales para determinar un abordaje terapéutico idóneo.

Hemos encontrado dos principales líneas de tratamiento. Con sus diferencias, los resultados funcionales han sido similares y comparables, sin desplazamiento cubital del carpo ni inestabilidad residual. La importancia de la fijación de la estiloides radial es un punto en común para garantizar la estabilidad ligamentaria.

Conflicto de intereses: Los autores no declaran conflictos de intereses.

ORCID de J. Parma: https://orcid.org/0000-0003-0337-289X

ORCID de E. Lombardo: https://orcid.org/0000-0003-3600-628X

ORCID de A. Barbero: https://orcid.org/0000-0002-8590-6383

ORCID de E. González: https://orcid.org/0000-0002-6109-1431
ORCID de J. Derico: https://orcid.org/0000-0002-2520-4128

ORCID de J. M. Baravalle: https://orcid.org/0000-0003-0337-641X

ORCID de M. Vélez: https://orcid.org/0000-0003-4094-0876

\section{BIBLIOGRAFÍA}

1. Dumontier C, Meyer zu Reckendorf G, Sautet A, Lenoble E, Saffar P, Allieu Y. Radiocarpal dislocations: classification and proposal for treatment. A review of twenty-seven cases. J Bone Joint Surg Am 2001;83(2):212-8. https://doi.org/10.2106/00004623-200102000-00008

2. Ilyas AM, Mugdal CS. Radiocarpal fracture-dislocations. J Am Acad Orthop Surg 2008;16(11):647-55. https://doi.org/10.5435/00124635-200811000-00005

3. Moneim MS, Bolger JT, Omer GE. Radiocarpal dislocation--classification and rationale for management. Clin Orthop Relat Res 1985;192:199-209. PMID: 39674323

4. Spiry C, Bacle G, Marteau E, Charruau B, Laulan J. Radiocarpal dislocations and fracture-dislocations: Injury types and long-term outcomes. Orthop Traumatol Surg Res 2018;104(2):261-6. https://doi.org/10.1016/j.otsr.2017.12.016

5. Girard J, Cassagnaud X, Maynou C. Radiocarpal dislocation: twelve cases and a review of the literature. Rev Chir Orthop Reparatrice Appar Mot 2004;90(5):426-33. https://doi.org/10.1016/s0035-1040(04)70169-5

6. Cornu A, Sturbois-Nachef N, Baudoux M, Amouyel T, Saab M, Chantelot C. Radiocarpal dislocation: a retrospective study of 14 patients. Orthop Traumatol Surg Res 2019;105(8):1611-6. https://doi.org/10.1016/j.otsr.2019.08.017

7. Potter MQ, Haller JM, Tyser AR. Ligamentous radiocarpal fracture-dislocation treated with wrist-spanning plate and volar ligament repair. J Wrist Surg 2014;3(4):265-8. https://doi.org/10.1055/s-0034-1394134 